\title{
Evaluation of Uniblends and Biblends of Commercial Maize Hybrids
}

\author{
K. Murali Krishna ${ }^{1 *}$, P. Laxminarayana ${ }^{2}$, G. K. Chikkappa ${ }^{3}$, P. Sambasiva Rao ${ }^{4}$, Kuldeep Singh Dangi ${ }^{5}$, J. P. Shahi ${ }^{6}$ and N. Kulkarni ${ }^{7}$ \\ ${ }^{1,5}$ Dept. of Genetics and Plant Breeding, 2Dept. of Agronomy, College of Agriculture, Rajendranagar, PJTSAU, Hyderabad \\ Telangana (500 030), India \\ ${ }^{3}$ Scientist, Plant Breeding, Indian Institute of Maize Research, Pusa Campus, New Delhi (110012), India \\ ${ }^{4}$ Acharya N. G. Ranga Agricultural University, Guntur, Andhra Pradesh (522 034), India \\ ${ }^{6}$ Dept. of Genetics and Plant Breeding, Institute of Agricultural Sciences, BHU, Varanasi, U.P. (221 005), India \\ ${ }^{7}$ Formely Dept. of Plant Breeding, PJTSAU, Rajendranagar, Hyderabad, Telangana (500 030), India
}

\section{Corresponding Author}

K. Murali Krishna

e-mail:kmurli73@yahoo.com

\author{
Article History \\ Article ID: 3 C0465 \\ Received in $10^{\text {th }}$ October, 2017 \\ Received in revised form $19^{\text {th }}$ December, 2017 \\ Accepted in final form 24 $4^{\text {th }}$ January, 2018
}

\begin{abstract}
Hybrid mixtures have been suggested as a mean to achieve increased crop productivity. Eight medium-season commercial maize (Zea mays L.) hybrids of public as well as private sectors were evaluated as uniblends as well as all possible biblends arrived by mixing equal quantity of seed in RCBD with three replications, during rabi, 2015 at Student's farm, College of Agriculture, Rajendranagar, Hyderabad. Data were recorded for grain yield and its components. Three uniblends viz., DHM-117, DHM-121 and SUNKRANTHI recorded highly significant positive general mixing ability effects for grain yield and the yield contributing characters. These uniblends can be used to develop high yielding hybrid mixture combinations and as a base population for further improvement. Among 28 combinations, four biblends viz., DHM117×SUN KRANTHI and DHM-121×SUN KRANTHI, GK-3153×NMH-731 and GK-3063×GK-3153 recorded high per se of 15.59-21.41 percent and expressed significant competitive ability over the high yielding uniblends. In general, the high yielding biblends involved at least one high yielding uniblend. Cross-pollination boost to seed yield was found to be greater from two hybrids which have different parentage or sources. Diallel analysis of mixing ability analogous to genetic analysis of combining ability demonstrated that uniblends differed in their ability to determine grain yield in biblends. The ability to predict biblend performance based on uniblend performance, together with the potential for above average grain yield, suggested that biblends can be formulated to achieve specific production requirements.
\end{abstract}

Keywords: Maize, mixing ability, blend, diallel and yield

\section{Introduction}

Maize (Zea mays L.) is one of the most important grain crops and plays a crucial role in global food security. Changing environmental conditions may increase the positive effects of mixtures of varieties/hybrids in terms of production and provision of ecosystem services. The current context of global environmental changes and increase in the frequency and severity of extreme climate events such as heat waves or drought (IPCC, 2013) may reinforce the positive effects of mixtures varieties. Two non-exclusive strategies can be proposed to detect the best variety combinations (Gaba et al., 2015) "blend screening" of a wide range of randomly assembled varieties vs trait-based approaches to choose interesting mixtures.

The trait-blend approach, and mixtures of varieties and their traits should foster complementary / facilitation and sampling effects to provide services and not only the yield.
Work done on mixtures in cross-pollinated crops like maize is meager and the pattern is less clear compared to selfpollinated crops. An understanding of the ways in which genotypes interact is essential for the perfect design and development of heterogeneous population, whether for breeding or production purposes. Useful information can be generated by studying the effects of blending two or more genotypes in simple mixtures. Superiority of certain mixtures over the mean of components and occasionally exceeded these of better performing component (Roy, 1965).

Mixtures have been reported to have more resistance to stress conditions than homogeneous cultivars. Mundt (2002) concluded that mixtures demonstrated greater yield stability than did the individual cultivars in case of wheat cultivars.

In this context, the present investigation was taken to evaluate the relative yield performance of maize hybrid mixtures as biblends over unblended. Analysis of general mixing ability 
(GMA) and specific mixing ability (SMA) assume importance as pre-requirite embarking on sound crop improvement program through genotype mixtures. Therefore, the present experiment was conducted in order to study about the mixing ability effects of selected genotypes of maize and identity desirable genotypes for synthesizing promising biblends mixtures.

\section{Materials and Methods}

Eight commercial maize hybrids viz., DHM-117, DHM-121, GK-3063, GK-3124, GK-3153, NMH-731, SUN KRANTHI and SUN GOLD were selected based on similar maturities and similar yield potentials. The hybrids were physically mixed in equal quantity to generate twenty eight biblends. Each biblend along with uniblend were grown in four row plot of four meters length with $75 \times 20 \mathrm{~cm}^{2}$ spacing in a randomized complete block design (RCBD) with three replications during winter season 2015 at student's farm, College of Agriculture, PJTSAU, Rajendranagar, Hyderabad. The trial was conducted in a sandy loam soil. All the recommended agronomic practices were followed to raise a good crop. Data were recorded on ten randomly selected plants in each treatment for nine characters viz., plant height $(\mathrm{cm})$, ear height, ear length $(\mathrm{cm})$, ear girth $(\mathrm{cm})$, number of kernel rows, number of kernels per row, 100 seed weight (g), shelling percent and grain yield hectare $^{-1}$. Data were analyzed treating the experiment as mechanical diallel following Griffing's Method 2 (Griffing 1956)
Model 1 that uses parents and crosses without reciprocals except that it allows for the partitioning of specific combining ability into variation attributable to (i) among parents (ii) parents vs crosses sources. Uniblends and biblends in this study were considered analogous to parents and crosses respectively, of diallel crosses used in genetic applications. This was justified by the fact that plants from crosses in genetic applications contain equal contributions of both parents, while each blend in this study contained equal contributions of its two uniblend components (Alam et al., 2012).

The plot means were subjected to mixing ability analysis in the same fashion as combining ability analysis of lines in hybrid combinations (Federer et al., 1982) using method 2, model 1 of Griffing (1956).

The term general mixing ability (GMA) was used to describe the average performance of a uniblend in biblends determined on the basis of the performance of biblends having that uniblend as a component. This term is similar in concept to general combining ability used in genetic applications. Similarly, the term specific mixing ability (SMA) was used to describe the deviation in performance of a mixture from that predicted by the GMA of both parents. This term is similar in concept to specific combining ability in genetic applications.

\section{Results and Discussion}

Based on per se performance (Table 1), the uniblend, $\mathrm{NMH}$ 731 recorded high mean for grain yield ha-1, plant height,

Table 1: Mean performance of uniblends and biblends of maize for yield and yield components

\begin{tabular}{|c|c|c|c|c|c|c|c|c|c|c|}
\hline $\begin{array}{l}\text { SI. } \\
\text { No. }\end{array}$ & Genotypes & $\begin{array}{c}\text { Plant } \\
\text { height } \\
(\mathrm{cm})\end{array}$ & $\begin{array}{c}\text { Ear } \\
\text { height } \\
(\mathrm{cm})\end{array}$ & $\begin{array}{c}\text { Ear } \\
\text { length } \\
(\mathrm{cm})\end{array}$ & $\begin{array}{l}\text { Ear } \\
\text { girth } \\
(\mathrm{cm})\end{array}$ & $\begin{array}{c}\text { Kernel } \\
\text { rows }\end{array}$ & $\begin{array}{l}\text { Kernels } \\
\text { /rows }\end{array}$ & $\begin{array}{c}100 \\
\text { Seed } \\
\text { weight } \\
\text { (g) }\end{array}$ & $\begin{array}{c}\text { Shelling } \\
\%\end{array}$ & $\begin{array}{c}\text { Grain } \\
\text { yield } \\
\text { ha }^{-1}(\mathrm{t})\end{array}$ \\
\hline 1. & DHM-117×DHM-121 & 162.75 & 77.05 & 16.6 & 14.55 & 14.45 & 30.9 & 23.685 & 83.1372 & 9.4999 \\
\hline 2. & DHM-117×GK-3063 & 143.2 & 66.8 & 16.3 & 13.7 & 14.25 & 30.85 & 24.6 & 81.3621 & 8.0833 \\
\hline 3. & DHM-117×GK-3153 & 174.6 & 82.2 & 15.6 & 13.3 & 14.85 & 27.55 & 24.69 & 93.9305 & 8.219 \\
\hline 4. & DHM-117×GK-3124 & 181.45 & 90.3 & 16.2 & 13.25 & 14.35 & 30.8 & 24.59 & 92.3911 & 8.4666 \\
\hline 5. & DHM-117×NMH-731 & 172.55 & 86.55 & 15.6 & 13.55 & 14.7 & 30.8 & 23.95 & 81.3721 & 7.4999 \\
\hline 6. & Dhm-117×Sun Kranthi & 167.5 & 83.05 & 16.7 & 14.1 & 15.1 & 33.15 & 26.54 & 96.0149 & 9.6832 \\
\hline 7. & DHM-117×Sun Gold & 167.5 & 76.65 & 16.3 & 13.6 & 15.3 & 33.75 & 26.295 & 96.0517 & 9.8332 \\
\hline 8. & DHM-121×GK-3063 & 160.2 & 76.3 & 16.2 & 12.85 & 14.3 & 29.4 & 24.155 & 92.2218 & 7.5499 \\
\hline 9. & DHM-121×GK-3153 & 157.9 & 74.65 & 15.55 & 13.05 & 15.25 & 30.35 & 24.22 & 81.3671 & 8.2833 \\
\hline 10. & DHM-121×GK-3124 & 156.75 & 75.8 & 16.55 & 13.05 & 14.6 & 30.65 & 22.25 & 95.3772 & 7.8499 \\
\hline 11. & DHM-121×NMH-731 & 153.1 & 75.4 & 16.25 & 13.2 & 15.1 & 30.85 & 24.305 & 79.9475 & 8.0999 \\
\hline 12. & Dhm-121×Sun Kranthi & 162.2 & 80.5 & 16.65 & 13.35 & 14.35 & 34.8 & 25.805 & 96.0239 & 9.5832 \\
\hline 13. & DHM-121×Sun Gold & 149.3 & 72.3 & 16 & 12.8 & 14.15 & 31.35 & 23.85 & 91.962 & 8.1265 \\
\hline 14. & GK-3063×GK-3153 & 156.55 & 74.95 & 16.55 & 12.95 & 13.9 & 34.3 & 25.78 & 88.1587 & 9.8166 \\
\hline 15. & GK-3063×GK-3124 & 155.25 & 76.1 & 17.35 & 13.3 & 15.2 & 33.6 & 23.605 & 96.0704 & 8.6332 \\
\hline 16. & GK-3063×NMH-731 & 156.2 & 82.65 & 15.4 & 13.15 & 15.3 & 30.15 & 24.455 & 87.388 & 6.1666 \\
\hline
\end{tabular}




\begin{tabular}{|c|c|c|c|c|c|c|c|c|c|c|}
\hline $\begin{array}{l}\text { Sl. } \\
\text { No }\end{array}$ & Genotypes & $\begin{array}{l}\text { Plant } \\
\text { height } \\
(\mathrm{cm})\end{array}$ & $\begin{array}{c}\text { Ear } \\
\text { height } \\
\text { (cm) }\end{array}$ & $\begin{array}{c}\text { Ear } \\
\text { length } \\
(\mathrm{cm})\end{array}$ & $\begin{array}{l}\text { Ear } \\
\text { girth } \\
(\mathrm{cm})\end{array}$ & $\begin{array}{l}\text { Kernel } \\
\text { rows }\end{array}$ & $\begin{array}{l}\text { Kernels } \\
\text { rows }^{-1}\end{array}$ & $\begin{array}{c}100 \\
\text { Seed } \\
\text { weight } \\
\text { (g) }\end{array}$ & $\begin{array}{c}\text { Shelling } \\
\%\end{array}$ & $\begin{array}{c}\text { Grain } \\
\text { yield } \\
\text { ha-1 }^{-1}(t)\end{array}$ \\
\hline 17. & Gk-3063×Sun Kranthi & 162.7 & 80.7 & 14.45 & 11.55 & 13.6 & 26.6 & 19.42 & 95.5882 & 8.2973 \\
\hline 18. & Gk-3063×Sun Gold & 165.9 & 80 & 15.8 & 12.75 & 14.5 & 29.4 & 23.84 & 83.1557 & 7.7499 \\
\hline 19. & GK-3153×GK-3124 & 150.75 & 72.75 & 16.4 & 13.25 & 14.5 & 33 & 20.265 & 83.1152 & 8.4666 \\
\hline 20. & GK-3153×NMH-731 & 155.5 & 76.6 & 16.5 & 13.1 & 14.8 & 33.9 & 23.515 & 79.782 & 9.3499 \\
\hline 21. & Gk-3153×Sun Kranthi & 145.5 & 70.9 & 15.55 & 12.85 & 15.4 & 33.05 & 21.36 & 83.149 & 7.7142 \\
\hline 22. & GK-3153×Sun Gold & 168.4 & 81.9 & 16.75 & 12.4 & 14.2 & 31.5 & 21.8 & 85.581 & 6.2149 \\
\hline 23. & GK-3124×NMH-731 & 148.95 & 74.4 & 17.05 & 13.55 & 14.5 & 32.05 & 23.85 & 96.0254 & 9.1666 \\
\hline 24. & Gk-3124×Sun Kranthi & 173.55 & 82.2 & 16.5 & 12.95 & 14.5 & 34 & 25.115 & 95.9364 & 9.7166 \\
\hline 25. & Gk-3124×Sun Gold & 176.05 & 83.5 & 15.5 & 13 & 13.2 & 31.15 & 23.95 & 79.8715 & 7.7835 \\
\hline 26. & Nmh-731×Sun Kranthi & 174.8 & 87.75 & 16.4 & 12.85 & 15.1 & 30.8 & 23.67 & 84.9115 & 7.5499 \\
\hline 27. & Nmh-731×Sun gold & 173.65 & 84.7 & 17.75 & 14.7 & 13.8 & 28.7 & 24.7 & 82.0835 & 7.6065 \\
\hline 28. & Sun kranthi×Sun gold & 159.35 & 75.9 & 15.75 & 13.25 & 14.85 & 29.15 & 23.78 & 76.7175 & 8.6666 \\
\hline 29. & DHM-117 & 169.6 & 80.65 & 16.05 & 13.35 & 15.6 & 29.6 & 25.1 & 95.7482 & 7.5833 \\
\hline 30. & DHM-121 & 161.3 & 79.75 & 16.8 & 12.75 & 13.95 & 30.65 & 23.98 & 96.0049 & 7.7499 \\
\hline 31. & GK-3063 & 161.75 & 78.5 & 16.275 & 12.9 & 14.6 & 31.9 & 23.8 & 94.5619 & 7.0625 \\
\hline 32. & GK-3153 & 165.2 & 80 & 15.6 & 12.5 & 14.7 & 31.75 & 20.105 & 95.8945 & 7.7166 \\
\hline 33. & GK-3124 & 162.6 & 80.1 & 17 & 13.3 & 14.6 & 29.15 & 24.16 & 82.0439 & 7.7666 \\
\hline 34. & $\mathrm{NMH}-731$ & 172.9 & 82.45 & 15.55 & 13.7 & 14.7 & 33.05 & 23.05 & 79.825 & 8.0833 \\
\hline 35. & Sun Kranthi & 154.2 & 76.4 & 15.65 & 12.9 & 14.2 & 29.2 & 25.125 & 84.5682 & 7.9666 \\
\hline \multirow[t]{5}{*}{36.} & Sun Gold & 149.3 & 71.4 & 14.85 & 13.05 & 13.5 & 28.85 & 22.545 & 86.5 & 7.9166 \\
\hline & Mean & 161.9153 & 78.6611 & 16.166 & 13.1778 & 14.5542 & 31.1306 & 23.7751 & 88.1622 & 8.2089 \\
\hline & C.V. & 3.9246 & 5.0503 & 3.6321 & 3.3094 & 2.942 & 4.9412 & 4.1563 & 0.2144 & 5.6663 \\
\hline & $\mathrm{CD}(p=0.05)$ & 10.3481 & 6.4693 & 0.9562 & 0.7102 & 0.6973 & 2.5049 & 1.6092 & 0.3077 & 0.7575 \\
\hline & $\mathrm{CD}(p=0.01)$ & 13.7386 & 8.5889 & 1.2695 & 0.9428 & 0.9257 & 3.3256 & 2.1364 & 0.4086 & 1.0056 \\
\hline
\end{tabular}

ear height, ear girth, number of kernel rows and number of kernels ear ${ }^{-1}$. The uniblend, SUN KRANTHI recorded high mean for grain yield ha-1 and 100 seed weight. The biblends, DHM-117×Sun Kranthi and DHM-121×Sun Kranth exhibited high per se for grain yield/hectare, number of kernels /row and 100 seed weight and whereas the biblend, GK-3063x GK-3153 recorded high mean value for grain yield hectare ${ }^{-1}$, ear length, number of kernels per row, 100 seed weight and shelling \%. The biblend, GK-3153 x NMH-731 for grain yield $\mathrm{ha}^{-1}$, ear length, ear girth, number of kernel rows and number of kernels row ${ }^{-1}$ and 100 seed weight exhibited high per se performance.

The genotypic differences for grain yield and its eight component characters were significant indicating the enough variation existed among the genotypes. Further partitioning of mixing ability revealed significant variation either due to general or due to specific mixing ability for all traits. However, general mixing ability (GMA) mean squares were highly significant for plant height, ear height, ear length, ear girth, number of kernels per row, 100 seed weight and grain yield $\mathrm{ha}^{1}$, whereas specific mixing ability was highly significant for grain yield $\mathrm{ha}^{-1}$, number of kernel rows and shelling percent (Table 2). The uniblend, DHM-117 has recorded highly significant positive general mixing ability effects for grain yield and the yield contributing characters like plant height, ear girth, 100 seed weight and shelling percent (Table 3). The uniblend, DHM-121 had recorded high per se and highly significant positive general mixing ability effects for grain yield ha ${ }^{-1}$, number of kernel row ${ }^{-1}$ and 100 seed weight whereas the uniblend Sun Kranthi recorded high per se and highly significant positive general mixing ability effects for plant height and number of kernels row ${ }^{-1}$. Hence, these uniblends can be used to develop high yielding hybrid mixture combinations and as a base population for further improvement. Alam et al. (2012) found that the genotype BD6893 in both sets performed best for yield per plant reflecting 


\begin{tabular}{|c|c|c|c|c|c|c|c|c|c|c|}
\hline & & $\begin{array}{l}\text { Plant } \\
\text { height } \\
(\mathrm{cm})\end{array}$ & $\begin{array}{l}\text { Ear height } \\
\quad(\mathrm{cm})\end{array}$ & $\begin{array}{l}\text { Ear } \\
\text { length } \\
(\mathrm{cm})\end{array}$ & $\begin{array}{l}\text { Ear girth } \\
(\mathrm{cm})\end{array}$ & $\begin{array}{l}\text { Kernel } \\
\text { rows }\end{array}$ & $\begin{array}{l}\text { Kernels } \\
\text { row }^{-1}\end{array}$ & $\begin{array}{c}100 \\
\text { Seed } \\
\text { weight } \\
(\mathrm{gm})\end{array}$ & Shelling \% & $\begin{array}{l}\text { Grain } \\
\text { yield/ } \\
\text { ha }^{-1}(\mathrm{t})\end{array}$ \\
\hline \multicolumn{2}{|c|}{ Replicates } & 56.375 & $244.922^{* * *}$ & ${ }^{*} \quad 21.739^{* * *}$ & *** $10.134^{* * *}$ & $\quad 3.706^{* * *}$ & $109.551^{* *}$ & $3.812^{*}$ & $1.73^{* * *}$ & $0.814^{*}$ \\
\hline \multicolumn{2}{|c|}{ Treatments } & $272.137^{* *}$ & $75.023^{* * *}$ & $1.367^{* * *}$ & ${ }^{*} \quad 0.948^{* * *}$ & $0.936^{* * *}$ & $11.679^{* * *}$ & $7.789^{* * *}$ & $131.475^{* * *}$ & $2.501^{* * *}$ \\
\hline \multicolumn{2}{|c|}{ Parents } & $215.14^{* * *}$ & $62.849^{* *}$ & $2.607^{* * *}$ & $1.992^{* * *}$ & $1.213^{* * *}$ & $6.636^{*}$ & $2.996^{* *}$ & $64.993^{* * *}$ & $3.717^{* * *}$ \\
\hline \multicolumn{2}{|c|}{ Hybrids } & $295.982^{* *}$ & $80.67^{* * *}$ & $1.089^{* * *}$ & $0.636^{* * *}$ & $0.891^{* * *}$ & $13.181^{* * *}$ & $9.252^{* * *}$ & $142.549^{* * *}$ & $1.86^{* * *}$ \\
\hline \multicolumn{2}{|c|}{$\begin{array}{l}\text { Parent Vs. } \\
\text { Hybrids }\end{array}$} & 27.322 & 7.757 & 0.175 & $2.081^{* * *}$ & 0.193 & 6.404 & 1.826 & $297.83^{* * *}$ & $11.285^{* * *}$ \\
\hline \multicolumn{2}{|c|}{ Error } & 40.38 & 15.782 & 0.345 & 0.19 & 0.183 & 2.366 & 0.976 & 0.036 & 0.216 \\
\hline \multicolumn{2}{|c|}{ Total } & 116.488 & 39.443 & 1.079 & 0.624 & 0.495 & 7.416 & 3.258 & 43.062 & 0.975 \\
\hline \multicolumn{2}{|c|}{ GCA } & $187.496^{* *}$ & $43.99^{* * *}$ & $0.533^{* * *}$ & $0.8^{* * *}$ & $0.185^{* * *}$ & $4.118^{* * *}$ & $3.788^{* * *}$ & $8.254^{* * *}$ & $0.925^{* * *}$ \\
\hline \multicolumn{2}{|c|}{ SCA } & $66.516^{* * *}$ & $20.262^{* * *}$ & $0.436^{* * *}$ & $0.195^{* * *}$ & $0.344^{* * *}$ & $3.837^{* * *}$ & $2.298^{* * *}$ & $52.718^{* * *}$ & $0.811^{* * *}$ \\
\hline \multicolumn{2}{|c|}{ GCA/SCA } & 2.81 & 2.17 & 1.22 & 4.1 & 0.537 & 1.073 & 1.648 & 0.156 & 1.14 \\
\hline \multicolumn{2}{|c|}{ Error } & 13.46 & 5.261 & 0.115 & 0.063 & 0.061 & 0.789 & 0.325 & 0.012 & 0.072 \\
\hline \multicolumn{2}{|r|}{ DF } & $\begin{array}{l}\text { Plant } \\
\text { height } \\
(\mathrm{cm})\end{array}$ & $\begin{array}{c}\text { Ear } \\
\text { height } \\
(\mathrm{cm}) \\
\end{array}$ & $\begin{array}{l}\text { Ear } \\
\text { length } \\
(\mathrm{cm}) \\
\end{array}$ & $\begin{array}{l}\text { Ear girth } \\
(\mathrm{cm})\end{array}$ & $\begin{array}{c}\text { Kernel } \\
\text { rows }\end{array}$ & $\begin{array}{c}\text { Kernels } \\
\text { row }^{-1}\end{array}$ & $\begin{array}{l}100 \text { Seed } \\
\text { weight } \\
(\mathrm{g})\end{array}$ & Shelling \% & $\begin{array}{c}\text { Grain } \\
\text { yield/ha-1 } \\
\text { (t) }\end{array}$ \\
\hline 1 & DHM-117 & $3.526^{* *}$ & 0.8 & 0.061 & $0.485^{* * *}$ & 0.076 & -0.208 & $0.821^{* * *}$ & $0.616^{* * *}$ & $0.445^{* * *}$ \\
\hline 2 & DHM-121 & $-6.509^{* * *}$ & $-3.68^{* * *}$ & 0.126 & -0.015 & 0.106 & $0.693^{*}$ & $0.466^{* *}$ & $-0.182^{* * *}$ & $0.288^{* * *}$ \\
\hline 3 & GK-3063 & $-3.314^{* *}$ & -0.37 & $-0.384^{* * *}$ & $-0.245^{* *}$ & $0.186^{*}$ & $-0.572^{*}$ & $-0.973^{* * *}$ & $-0.459^{* * *}$ & $-0.39^{* * *}$ \\
\hline 4 & GK-3153 & $5.016^{* * *}$ & $3.01^{* * *}$ & 0.036 & $-0.345^{* * *}$ & $-0.224^{* *}$ & -0.093 & $-0.548^{* *}$ & $0.237^{* * *}$ & $-0.237^{* *}$ \\
\hline 5 & GK-3124 & $2.991^{* *}$ & $1.92^{* *}$ & $0.371^{* * *}$ & $0.335^{* * *}$ & -0.094 & $-0.633^{*}$ & $0.573^{* *}$ & $-0.425^{* * *}$ & -0.067 \\
\hline 6 & NMH-731 & -0.549 & -0.465 & 0.101 & -0.065 & 0.061 & 0.517 & -0.256 & $1.735^{* * *}$ & 0.039 \\
\hline 7 & $\begin{array}{c}\text { SUN } \\
\text { KRANTHI }\end{array}$ & $3.316^{* *}$ & 0.57 & $-0.224^{*}$ & 0.02 & 0.011 & $0.958^{* * *}$ & 0.199 & $-1.374^{* * *}$ & $0.234^{* *}$ \\
\hline \multirow[t]{2}{*}{8} & $\begin{array}{l}\text { SUN } \\
\text { GOLD }\end{array}$ & $-4.479^{* * *}$ & $-1.785^{*}$ & -0.084 & $-0.17^{*}$ & -0.124 & $-0.663^{*}$ & -0.283 & $-0.149^{* * *}$ & $-0.312^{* * *}$ \\
\hline & $\mathrm{Gi}$ & 2.56 & 1.60 & 0.23 & 0.17 & 0.17 & 0.62 & 0.39 & 0.07 & 0.18 \\
\hline
\end{tabular}

the best uniblend genotype in mung bean ( Vigna radiate L.). The specific mixing ability effects are highly valuable for discrimination of biblends for their yielding ability. Among the 28 biblends, nine combinations exhibited significant positive specific mixing effects for yield (Table 4). In the biblend, DHM$121 \times$ SUN KRANTHI, both the parents, exhibited high per se and significant general mixing ability for grain yield. Kannenberg and Hunter (1972) opined that high yielding hybrids contributed more than would be expected relative to their pure stand yield and low yielding hybrids less than expected. Springfield (1958) opined that mixtures of either similar or dissimilar hybrids had any clear advantage or disadvantage when compared with averages of the contributing hybrids grown separately. On the contrary, Alam et al.,(2012) reported that the biblend mixture BD-6901+BD-6922 showed the best performance for yield plant $^{-1}$ in mung bean (Vigna radiate L.), Mark (1997) reported about a four bushel average gain in yield from mixing maize hybrids developed from different seed companies. In this investigation also, the biblend, DHM$117 \times$ Sun Kranthi exhibited significant positive SMA effect for grain yield hectare ${ }^{-1}$, number of kernel rows, number of kernels/ row, 100 seed weight and shelling percent. The biblend, DHM-121xSUN KRANTHI recorded significant positive SMA for grain yield ha- ${ }^{-1}, 100$ seed weight and shelling $\%$. The biblend, GK-3153×NMH-731 exhibited significant positive SMA for grain yield ha-1 ${ }^{-1}$ plant height, number of kernel rows, 100 


\begin{tabular}{|c|c|c|c|c|c|c|c|c|c|c|}
\hline $\begin{array}{l}\text { Sl. } \\
\text { No. }\end{array}$ & Genotypes & $\begin{array}{l}\text { Plant } \\
\text { height } \\
(\mathrm{cm})\end{array}$ & $\begin{array}{l}\text { Ear } \\
\text { height } \\
(\mathrm{cm})\end{array}$ & $\begin{array}{l}\text { Ear } \\
\text { length } \\
(\mathrm{cm})\end{array}$ & $\begin{array}{l}\text { Ear } \\
\text { girth } \\
(\mathrm{cm})\end{array}$ & $\begin{array}{l}\text { Kernel } \\
\text { rows }\end{array}$ & $\begin{array}{c}\text { Kernels / } \\
\text { rows }\end{array}$ & $\begin{array}{c}100 \text { Seed } \\
\text { weight } \\
\text { (g) }\end{array}$ & Shelling \% & $\begin{array}{c}\text { Grain } \\
\text { yield ha-1 } \\
\text { (t) }\end{array}$ \\
\hline 1. & $\begin{array}{l}\text { DHM- } \\
117 \times \text { DHM-121 }\end{array}$ & $-15.733^{* * *}$ & $-8.981^{* * *}$ & -0.052 & 0.052 & $-0.487^{*}$ & -0.766 & -0.462 & $-7.234^{* * *}$ & $-0.859^{* *}$ \\
\hline 2. & $\begin{array}{l}\text { DHM- } \\
117 \times \text { GK-3063 }\end{array}$ & $12.472^{* * *}$ & 3.109 & -0.242 & -0.118 & 0.033 & $-2.801^{* *}$ & $1.066^{*}$ & $5.611^{* * *}$ & -0.045 \\
\hline 3. & $\begin{array}{l}\text { DHM-117x } \\
\text { GK-3153 }\end{array}$ & $10.992^{* *}$ & $7.829^{* * *}$ & -0.062 & -0.068 & -0.057 & -0.031 & 0.541 & $3.376^{* * *}$ & 0.049 \\
\hline 4. & $\begin{array}{l}\text { DHM-117x } \\
\text { GK-3124 }\end{array}$ & 4.117 & $5.169^{*}$ & $-0.997^{* *}$ & -0.448 & 0.163 & 0.509 & $-1.219^{*}$ & $-6.981^{* * *}$ & $-1.088^{* * *}$ \\
\hline 5. & $\begin{array}{l}\text { DHM-117x } \\
\text { NMH-731 }\end{array}$ & 2.607 & 4.054 & 0.373 & $0.502^{*}$ & 0.408 & $1.709^{*}$ & $2.200^{* * *}$ & $5.502^{* * *}$ & $0.99^{* * *}$ \\
\hline 6. & $\begin{array}{l}\text { Dhm-117x } \\
\text { Sun Kranthi }\end{array}$ & -1.258 & -3.381 & 0.298 & -0.083 & $0.658^{* *}$ & $1.869^{*}$ & $1.499^{* *}$ & $8.647^{* * *}$ & $0.945^{* * *}$ \\
\hline 7. & $\begin{array}{l}\text { DHM-117x } \\
\text { Sun Gold }\end{array}$ & -0.763 & -1.376 & 0.058 & $-0.643^{* *}$ & -0.207 & -0.861 & -0.158 & $3.593^{* * *}$ & $-0.793^{* *}$ \\
\hline 8. & $\begin{array}{l}\text { DHM-121x } \\
\text { GK-3063 }\end{array}$ & 4.657 & 1.189 & $0.643^{*}$ & 0.132 & -0.247 & -0.601 & -1.018 & $7.856^{* * *}$ & -0.257 \\
\hline 9. & $\begin{array}{l}\text { DHM-121x } \\
\text { GK-3153 }\end{array}$ & $-7.323^{*}$ & -2.591 & -0.077 & 0.382 & $0.663^{* *}$ & -0.881 & 0.612 & $-8.27^{* * *}$ & -0.16 \\
\hline 10. & $\begin{array}{l}\text { DHM- } \\
121 \times G K-3124\end{array}$ & 3.802 & 3.599 & -0.012 & -0.148 & -0.217 & $3.609^{* * *}$ & 0.991 & $8.469^{* * *}$ & $1.153^{* * *}$ \\
\hline 11. & $\begin{array}{l}\text { DHM-121× } \\
\text { NMH-731 }\end{array}$ & -5.558 & -2.216 & -0.392 & -0.298 & $-0.572^{*}$ & -0.991 & -0.135 & $2.247^{* * *}$ & -0.409 \\
\hline 12. & $\begin{array}{l}\text { Dhm-121×Sun } \\
\text { Kranthi }\end{array}$ & -2.173 & -0.601 & 0.483 & -0.233 & $-0.772^{* *}$ & 1.519 & $1.340^{*}$ & $1.552^{* * *}$ & $1.086^{* * *}$ \\
\hline 13. & $\begin{array}{l}\text { DHM-121×Sun } \\
\text { Gold }\end{array}$ & 4.322 & 2.904 & $1.143^{* * *}$ & 0.307 & $0.663^{* *}$ & $2.439^{* *}$ & -0.353 & $8.24^{* * *}$ & 0.448 \\
\hline 14. & $\begin{array}{l}\text { GK-3063× GK- } \\
3153\end{array}$ & -0.918 & -0.601 & $-1.367^{* * *}$ & $-1.038^{* *}$ & $-0.917^{* * *}$ & $-3.866^{* * *}$ & $-2.835^{* * *}$ & $7.647^{* * *}$ & $0.715^{* *}$ \\
\hline 15. & $\begin{array}{l}\text { GK-3063× GK- } \\
3124\end{array}$ & 4.307 & -0.211 & -0.352 & $-0.518^{*}$ & -0.147 & 0.526 & 0.465 & $-4.123^{* * *}$ & -0.002 \\
\hline 16. & $\begin{array}{l}\text { GK-3063x } \\
\text { NMH-731 }\end{array}$ & $-7.303^{*}$ & -5.076 & 0.518 & 0.382 & -0.302 & $1.924^{*}$ & $-2.281^{* * *}$ & $-6.323^{* * *}$ & $0.609^{*}$ \\
\hline 17. & $\begin{array}{l}\text { Gk-3063×Sun } \\
\text { Kranthi }\end{array}$ & -6.418 & -2.261 & $0.943^{* *}$ & 0.147 & 0.048 & $2.384^{* *}$ & 0.513 & $-6.548^{* * *}$ & $1.297^{* * *}$ \\
\hline 18. & $\begin{array}{l}\text { Gk-3063×Sun } \\
\text { Gold }\end{array}$ & $-8.623^{*}$ & $-5.606^{*}$ & -0.147 & 0.087 & $0.783^{* *}$ & $3.154^{* * *}$ & $-1.159^{*}$ & $-4.406^{* * *}$ & 0.207 \\
\hline 19. & $\begin{array}{l}\text { GK-3153× GK- } \\
3124\end{array}$ & $-20.973^{* * *}$ & $-9.191^{* * *}$ & 0.478 & 0.382 & 0.263 & 1.644 & 0.05 & $8.05^{* * *}$ & $1.261^{* * *}$ \\
\hline 20. & $\begin{array}{l}\text { G K - } 3153 \times \\
\text { NMH-731 }\end{array}$ & $7.167^{*}$ & 0.994 & 0.198 & 0.182 & 0.108 & $2.444^{* *}$ & $2.144^{* * *}$ & $5.502^{* * *}$ & $1.706^{* * *}$ \\
\hline 21. & $\begin{array}{l}\text { GK-3153×Sun } \\
\text { Kranthi }\end{array}$ & 5.802 & 1.259 & -0.477 & 0.147 & $-1.142^{* *}$ & -0.846 & 0.523 & $-7.155^{* * *}$ & -0.422 \\
\hline
\end{tabular}




\begin{tabular}{|c|c|c|c|c|c|c|c|c|c|c|}
\hline $\begin{array}{l}\text { Sl. } \\
\text { No. }\end{array}$ & Genotypes & $\begin{array}{l}\text { Plant } \\
\text { height } \\
(\mathrm{cm})\end{array}$ & $\begin{array}{c}\text { Ear } \\
\text { height } \\
(\mathrm{cm})\end{array}$ & $\begin{array}{l}\text { Ear } \\
\text { length } \\
(\mathrm{cm})\end{array}$ & $\begin{array}{l}\text { Ear girth } \\
(\mathrm{cm})\end{array}$ & $\begin{array}{l}\text { Kernel } \\
\text { rows }\end{array}$ & $\begin{array}{l}\text { Kernels } \\
\text { rows }^{-1}\end{array}$ & $\begin{array}{c}100 \text { Seed } \\
\text { weight } \\
\text { (g) }\end{array}$ & Shelling \% & $\begin{array}{c}\text { Grain } \\
\text { yield } \\
\mathrm{ha}^{-1}(\mathrm{t})\end{array}$ \\
\hline 22. & $\begin{array}{l}\text { GK-3153×Sun } \\
\text { Gold }\end{array}$ & $12.347^{* * *}$ & $7.864^{*}$ & 0.283 & 0.187 & $0.893^{* * *}$ & 0.424 & 0.726 & $-3.339^{* * *}$ & -0.11 \\
\hline 23. & $\begin{array}{l}\text { GK-3124× NMH- } \\
731\end{array}$ & -5.008 & -4.216 & $-0.887^{* *}$ & -0.198 & 0.328 & $-1.866^{*}$ & -0.312 & $-12.755^{* * *}$ & 0.486 \\
\hline 24. & $\begin{array}{l}\text { Gk-3124×Sun } \\
\text { Kranthi }\end{array}$ & 1.377 & -0.501 & -0.262 & -0.183 & $1.128^{* * *}$ & $-1.856^{*}$ & 0.553 & $9.384^{* * *}$ & $-0.792^{* *}$ \\
\hline 25. & $\begin{array}{l}\text { Gk-3124×Sun } \\
\text { Gold }\end{array}$ & 0.872 & 0.954 & 0.348 & $-0.593^{*}$ & -0.387 & 0.814 & -0.085 & $8.416^{* * *}$ & -0.081 \\
\hline 26. & $\begin{array}{l}\text { Nmh-731×Sun } \\
\text { Kranthi }\end{array}$ & 0.517 & 1.234 & -0.412 & $-0.633^{* *}$ & 0.073 & -0.856 & $-3.613^{* * *}$ & $7.371^{* * *}$ & $-0.765^{* *}$ \\
\hline 27. & $\begin{array}{l}\text { Nmh-731×sun } \\
\text { gold }\end{array}$ & 5.712 & 3.689 & $0.818^{*}$ & 0.357 & 0.108 & $-1.836^{*}$ & 0.924 & $-7.704^{* * *}$ & 0.169 \\
\hline 28. & $\begin{array}{l}\text { Sun kranthixsun } \\
\text { gold }\end{array}$ & -6.553 & -1.046 & -0.207 & -0.128 & -0.242 & $-2.226^{* *}$ & $1.434^{*}$ & $-2.071^{* * *}$ & -0.164 \\
\hline & Sij & 6.86 & 4.26 & 0.63 & 0.46 & 0.46 & 1.65 & 1.06 & 0.20 & 0.50 \\
\hline
\end{tabular}

seed weight and shelling \%. The biblend, GK-3063 x GK-3153 expressed significant positive SMA for grain yield hectare ${ }^{-1}$ and shelling \%. Thus, biblends of DHM-117×SUN KRANTHI, DHM$121 \times$ Sun Kranthi, GK-3153 x NMH-731 and GK-3063×GK-3153 gave higher yield of $15.59-21.41 \%$ over high yielding sole crop. The two hybrids in biblends may utilize the resources viz. light, nutrients and water in patterns that complement each other. The increase in the grain yield may be attributed to synergetic and compatibility with respect to wider adaptability to soil and weather conditions. This suggests the cross-pollination boost to seed yield is greater from two hybrids and would be more likely when hybrids collected from different sources.

Mumaw and Weber (1957) have obtained significant differences $(+2.1 \%)$ for the yield of 20 paired mixtures of soybean varieties over their average performance in pure stand with the superiority for mixed stands ranging from $-2.2 \%$ to $+6.3 \%$ for individual mixtures. Similarly in rice, Kulkarni (1995) reported $12 \%$ superiority of the varietal mixture combinations over sole varieties. Roy (1965) has emphasized that one has to make choice between purity and productivity. Some basic demands like flowering, plant height for uniformity must be satisfied while selecting the hybrid for mixture combinations. This will give a balance between purity and also productivity. As the hybrids chosen for the study are commercially released hybrids, the seed production for the hybrids will not be a bottleneck and the farmer will use his discretion in making the biblends based on the technology.

\section{Conclusion}

Thirty six genotypes comprising of twenty eight biblends and eight uniblends tested at Rajendranagar, Hyderabad during winter, 2015. Four hybrid mixtures combinations viz., DHM-117×SUN KRANTHI, DHM-121×Sun Kranthi, GK$3153 \times N M H-731$ and GK-3063×GK-3153 have exhibited significant positive specific mixing effects for grain yield recording $15.59-21.41 \%$ higher yield over their uniblends. Further evaluation with more combinations of biblends to identify high yielding biblends would be rewarding.

\section{References}

Alam, M.A., Mahmud, F., Uddin, S., Jahan, N., Asad, U., 2012. Mixing ability analysis of $7 \times 7$ uniblends and biblends of mung bean (Vigna radiata L.) genotypes. Bulletin of the Institute of Tropical Agriculture, Kyushu University $35,11-18$.

Doney, D.L., Plaisted, R. L., Peterson, L. C., 1965. Genotypic competition in progeny performance evaluation of potatoes. Crop Science 5, 433-435.

Eberhart, S.A., Penny, L. H., Sprague, G.F., 1964. Intraplot competition among maize single crosses. Crop science 4, 467-471.

Federer, W.T., Connigate, J.C., Rutger, J.N., Wijesinha, A., 1982. Statistical analyses of yields from uniblends and biblends of eight dry bean cultivars. Crop Science, 22: 111-115.

Funk, C.R., Anderson, J.C., 1964. Performance of mixtures of field corn (Zea mays L.) hybrids. Crop Science 4, 353-356.

Gaba, S., Lescurrent, F., Boudsocq, S., Enjalbert, J., Hinsinger P., 2015. Multiple cropping systems as drivers for providing multiple ecosystem service: from concepts to design. Agro sustainable Development 35(2), 607-623.

Greg Stewart., 2006. Corn hybrid mixing. GOCorn.net.

Griffing, B., 1956. Concepts of general and specific combining ability in relation to diallel crossing systems. Australian 
Journal of Biological sciences 9, 463-493.

IPCC (2013) - Climate change. 2013. The physical science basis. Contribution of working group I to the fifth assessment report of the intergovennatal panel on climate change Cambridge University Press, Cambridge United Kingdom and Newyork, NY, USA.

Kannenberg, L.W., Hunter, R.B., 1972. Yielding ability and competitive influence in hybrid mixtures of maize. Crop Science 12, 274-276.

Kulkarni, N., 1995. Competitive ability of medium duration rice for grain yield. IRRI News letter 21(1), 1.

Mark Westgate., 1997. GoCorn.net. Growing Ontario's corn.
Mumaw, C.R., Weber, C.R., 1957. Competition and natural selection in soybean varietal composites. Agronomy Journal 49, 154-160.

Mundt, C.C., 2002. Performance of wheat cultivars and cultivar mixtures in the presence of Cephalosporium stripe. Crop Protection 21(2), 93-99.

Roy, S.K., 1965. The Price of monoculture. Proceedings of $3^{\text {rd }}$ international symposium, 1995. Indian Society of Genetics and Plant Breeding, New Delhi.

Springfield, G.H., 1959. Performance of corn hybrids in mixtures. Agronomy Journal 51, 472-473. 